\section{Disaster Healthcare System Management and Crisis Intervention Leadership in Thailand-Lessons from the 26 December 2004 Tsunami Disaster}

Y. Bar-Dayan; ${ }^{1}$ N. Guy, ${ }^{2}$ A. Issac, ${ }^{3}$ S. Ofer, ${ }^{3}$ D. Shvarts; ${ }^{4}$

P. Rami; ${ }^{2}$ Y. Levi; ${ }^{3}$ A. Goldberg ${ }^{4}$

1. Faculty of Health Sciences, Israel

2. IDF Home Front Command, Israel

3. IDF Medical Corps, Surgeon General Headquarters, Israel

4. Faculty of Health Sciences, Ben Gurion University, Israel

Introduction: On 26 December 2004 at 09:00 hours, an earthquake with a magnitude of 9.0 on the Richter scale struck the area off the western coast of northern Sumatra, triggering massive tidal waves (tsunami). The tsunami waves flooded costal areas in countries around the Indian Ocean rim, including Thailand, causing a huge number of fatalities and injuries as well as destruction of infrastructure. In Thailand, on 25 January 2005, 5.388 fatal cases were confirmed, 3,120 people were reported missing, and 8,457 people were wounded. In disaster management research literature, there are several well-established criteria set up by Quarantelli for evaluating the effectiveness of disaster management.

Objectives: The aims of this study were to: (1) describe how the Thai health system managed the disaster which occurred in the wake of the tsunami; and (2) analyze the Thai response with reference to the Quarantelli principles. Method: The IDF Home Front Command Medical Department sent a research team to study the response of the Thai medical system in to the disaster. The delegation included three physicians and a behavioral psychologist experienced in hospital preparedness for disaster, and emergency medicine. The delegation arrived in Thailand one month after the tsunami. The team met with Thai healthcare officials including the General Director of the Ministry of Health and three Provincial Directors (in the areas affected by the tsunami). The team also met with the head of the Thai Air Force and visited six public hospitals and four community clinics involved in the care for the victims. Data collection was primarily through personal and group interviews. The analysis of the disaster management was based on Quarantelli's 10 criteria for evaluating the management of community disasters (1997).

Results: The criteria for the development of overall coordination were very significant in managing the operation, especially in the cases of the "war room" that conducted the operation in Krabi. The coordination of foreign volunteers, volunteers from unaffected areas in Thailand, and local workers was crucial. Leadership at different levels (Health Minister, provincial Governors, provincial doctors, and hospital managers) strongly affected the way the operation was managed. The criteria of carrying out generic functions in an adequate way were implemented partly in the tsunami disaster even though no one was prepared for it. Four factors are highly important in managing a mass-casualty disaster, even when local organizations never have planned for or experienced anything similar: The coordination and integration of all relevant organizations, the dominant leadership and management, the coordination between new health personnel (especially volunteers) and local staff, the communication, and processing of information are essential.

Conclusions: These principals seemed to be very relevant in analyzing the Thai health system's response to the masscasualty event caused by the tsunami waves. The implications, as Quarantelli pointed out, are that we should focus both on managing and not only on planning. This does not negate the need to prepare for disasters on a local, national, or even multi-national scale. Such preparation is vital to integrating strategy (planning) and tactics (management). Keywords: coordination; communication; criteria; disaster; effectiveness; healthcare system; information; leadership; management; Quarantelli; tsunami

Prehosp Disast Med 2005;20(3):s150

Managing a Mass-Casualty Incident in Phi Phi Island after the Tsunami on 26 December 2004-A Ruined Hospital with Hundreds of Injured Survivors Y. Bar-Dayan, ${ }^{1}$ S. Ammartyotbin, ${ }^{2}$ I. Ashkenasi ${ }^{3}$

1. IDF Home Front Command, Israel

2. Head of Phi Phi Island Hospital, Thailand

3. IDF Medical Corps, Surgeon General Hospital, Israel

Introduction: On 26 December 2004 at 09:00 hours, an earthquake with a magnitude of 9.0 on the Richter scale struck the area off the western side of northern Sumatra, triggering massive tidal waves (tsunami). The tsunami inundated costal areas in countries all around the Indian Ocean rim including Thailand, causing a huge number of fatalities and injuries as well as destruction of infrastructures. In Thailand, on 25 January 2005, 5,388 fatal cases were confirmed, 3,120 people were reported missing, and 8,457 people were wounded.

Objective: The aim of this article was to describe the rare experience of a young physician dealing for the first time with a disaster.

Method: The IDF Home Front Command Medical Department sent a research team to study the responses of the Thai medical system to the disaster. The team included three physicians and a behavioral sociologist experienced in hospital preparedness for disaster and emergency medicine. The delegation met with Thai healthcare officials and met and questioned other medical system managers and the hospital team of Phi Phi Island. The hospital was ruined in the tsunami and one of the staff members was killed and another one injured. The remaining staff survived to treat hundreds of patients in Phi Phi Island for 24 hours.

Results: The wave washed Phi Phi Island at 10:00 hours. The hospital was flooded by the wave and the team moved to the second floor and waited for two hours to be rescued from the flood. After two hours, the wave retreated and the physician and the nurses went out of the hospital and localized in the cabana hotel where they triaged 700 patients with one doctor and two nurses. The treatment priorities were changed-the main goal of the team was wound banding and external bleeding control. No A or B treatment was performed. The lying patients were transferred to 